Bemejlungen ïber unendichdimensionale, separierte Linesveltorräume und Iinesgruppen.

von K. Kutzler

Niv. 6

$(1971)$

Diese Arbeit entstand aus einem Teil der Dissertation des Verfassers. 
Bemerkungen uber unendjichaimensionales separierte Limes-

\section{vektorräume und Linespruppen.}

von Kurt Kutzler

Im Folgenden sej $K$ stets der Körper der reelien oder der komplexen Zahlen. Die Dimension eines Vektorraumes $V$ über $K$ sej definiert durch die Kardinalzahl einer (Hamel-) Basjs von $V$.

Für zwei Limitierungen $\tau_{1}$ und $\tau_{2}$ auf einer Menge $E$ gelte $\tau_{1} \geqq \tau_{2}$ genau dann, wenn die identische Abbildung. $\operatorname{id}_{\mathrm{E}}:\left(E, \tau_{1}\right) \longrightarrow\left(E, \tau_{2}\right)$ stetig ist. $\tau_{1}$ heißt feiner als. $\tau_{2}$ bzw. $\tau_{2}$ heiBt gröber als $\tau_{1}$. Das erste Ziel dieser Arbeit ist es, alif jedem unendichdimensionalen K-Vektorraum V eine bezügljch $\geqq$ nach unten gerichtete Familie von lokalkonvexen, separierten Vektorraumtopologien auf $V$ zu konstruieren, deren Mächtigkeit abzählbar ist und die zudem die Eigenschaft besitzt, daß eine Vektorraumtopologie auf $V$, ob lokalkonvex oder nicht, die gröber als jede Topologie der Familie ist, cie indiskrete Topologie auf $V$ sein muß. In anderen worten: Zu V wird ein ........ induktives System lokalkonvexer, separierter, topologischer vektorräume mit den folgenden Eigenschaften konstruiert:

(a) Für jedes Element des induktiven systems ist der unterliegende vektorraum mit $V$ identisch.

(b) Die Mächtigkeit des induktiven Systems ist abzählbar.

(c) Der induktive Limes dieses Systems in der Kategorje 
der topologischen und somit erst recht in der Kategorie der lokalkonvexen, topologischen Vektorräume ist $V$ versehen mit der indiskreten topologie. In der Kategorie der Limesvektorräume hingegen ist der induktive Limes dieses systems separiert.

Ein weiteres Ziel dieser Arbeit ist es; die Richtigkeit des Satzes von Fischer ([3], p. 294, Satz 6. ), daß auf jeder Limesgruppe dje zur Gruppenlimitierung nächstgröbere Topologie mit der Gruppenstruktur verträglich sei, zu überprüfen. Es waren von Frölicher und Bucher [4] Zweifel über die Richtigkeit des Satzes angedeutet worden. Bucher wies in [2] nach, daß der Beweis von Fischer falsch ist. Es soll hier nun auch nachgewiesen werden, daß der Satz selbst falscr. ist.Dies wird mit Hilfe der oben angedeuteten Mittel geschehen.

Beachtet man, daß der nach (a) - (c) zu definierende Limesvektorraum ein Marinescu-Raum im Sinne von H. Jarchow ([5], [6]) ist, so erhält man gleichzeitig ein sehr pathologisches Beispiel fü eine Marinescu--Iimitierung auf einem beliebigen unendichdimensionalen viktorraum.

Es war vom Verf. in einer früheren Note [8] gezeigt worden, daß die endichdimensionalen Vektorräume dje Eigenschaft besitzen, nur eine separierte Vektorraumlimitierung zuzulassen, nämlich die natürliche Topologie. In diesem Zusammenhang erkennt man, daß dịese Eigenschaft f'ï endichdimensionale K-Vektorrame charakteristisch ist. Für separierte, topolo- 
gische Vektorräume charakterisjert die Lokalkompaktheit des Vektorraumes auch dessen endiche Dimension, d.h., die endichaimsnsionalen, separierten topologischen vektorräume stimmen genau mit den lokalkompakten, separierten, topologigchen Vektorräumen überein. Nun ist der Begriff der Lokalikompaktheit im Zusammenhang mit Untersuchungen über Algebren stetiger, reellwertiger Funktionen auf Limesräumen von F.T.M.Schroder für Limesräume verallgemeinert worden. In diesem Zusammenhang ist es von Interesse zu wissen, ob das Analogon des soeben zitierten Charakterisierungssatzes endichdimensionaler, separierter, topologischer Vektorräume auch für separierte Limesvektorräume oder wenigstens für Marinescu-Räume gilt. Wiederum wird anhand einer speziellen Marinescu-Limitierung gezeigt werden, daß auf jedem unendlichdjmensionalen Vektorraum $V$. eine Limitierung $\tau$ derart existiert, daß $(V, \tau)$ ein separierter, lokalkompakter Marinescu-Raum ist. Das bedeutet, daß diese Charakterisierung endichdimensionaler Vektorräume kaum zu verallgemeinern ist.

\section{Begriffe.}

Im Folgenden seien:

(1.1) $L$ die Kategorie der Limesräume mit den stetigen Abbildungen als Morphismen.

(1.2) $T$ die Kategorie der topologischen Räume mit den stetiger Abbilangen als Morphismen. Bemerkung: $T$ ist aufgrund der Axiomatik der Iimesräume eine volle Unterkategorie von $L$. 
(1.3) Ds sei LU die Kategorie der Iimesvertorräume über dem Körper $K$.

(1.4) TV sei dje Kategorie der topologjschen Vektorräume über $K$.

(1.5) KV sei die Kategorie der lokalkonvexen, topglom gischen Vektorräume über $K$.

In diesen Kategorien seien jeweils die stetigen, Iinearen Abbildungen die Morphismen.

Bemerkung: $T V$ und $K V$ sind volle Unterkategorien von $L V$. Von Fischer [3] wurden die Begriffe der nächstgröberen Topologie für eine Limitierung und der nächstgröberen lokalkovexen Topologie für eine Vektorraumlimitierung eingeführt:

(1.6) Sei $(E, \tau)$ ein Limesraum. Dann gibt es eine eindeutig bestimmte Topologie wT auf $E$ derart, daß für jeden topologischen Raum $(F, \Lambda)$ und jede stetige Abbildling $f:(E, T) \longrightarrow(F, \Lambda)$ das folgende Diagram in $L$ kommutiert:

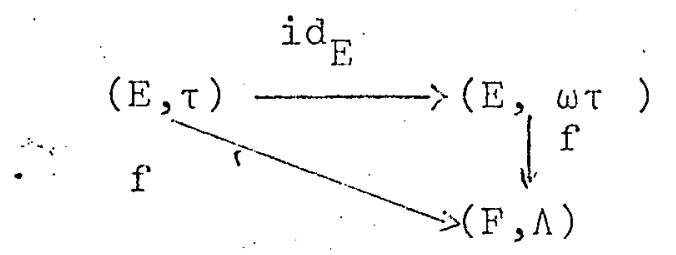

$\omega \tau$ wird die zu $\tau$ nächstgröbere Topologie genannt. Ist $f:(E, \tau) \longrightarrow\left(F, \tau^{\prime}\right)$ ein Morphismus aus $L$, so ist $f:(E, \omega \tau) \longrightarrow\left(E, \omega \tau^{\prime}\right)$ aus $T$. Man kann demnach die Operation $\omega$, die jeder Limitierung dje nächstgröbere Topologie zuordnet, als einen Funktor $\omega: L \longrightarrow T$ interpretieren, der definiert, ist durch

$$
\omega(E, \tau)=(E, \omega \tau)
$$


fü jeden Jimesraum $(E, \tau)$ und

$$
\omega\left(f:(E, \tau) \longrightarrow\left(F, \tau^{\prime}\right)\right)=f:(E, \omega \tau) \longrightarrow\left(F, \omega \tau^{\prime}\right)
$$

für jeden Morphismus $f:(E, \tau) \longrightarrow\left(E, \tau^{\prime}\right)$ aus $L$.

Bemerkung: Fischer [3] hat bewiesen, dab $\tau$ genau dann dem ersten Trennungsaxiom genügt, wenn die Topologie wi...tem ersten I'rennungsaxjom genügt.

(1.7) Sei (E, T) ein Limesvektorraum. Dann gibt es ejne (eindeutig bestimmte) lokalkonvexe Topologie $\psi^{\circ} \tau$ auf E dergestalt, daß für jeden lokalkonvexen, topologischen Vektorraum $(F, \Lambda)$ und für jede stetige, lineare Abbildung $f:(E, \tau) \longrightarrow(F, \Lambda)$ das folgende Diagramm in LV kommutiert:

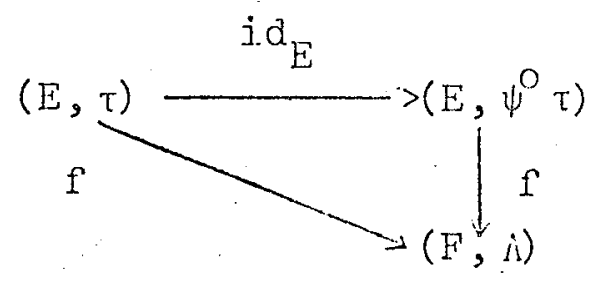

Sind $(E, \tau)$ und $\left(F, \tau^{\dagger}\right)$ zwei Limesvektorräume und ist $f:(E, \tau) \longrightarrow\left(F, \tau^{\prime}\right)$ linear und stetig, so ist auch die Abbildune $f:\left(E, \psi^{O} \tau\right) \longrightarrow\left(F_{s:}^{\prime} \psi^{O} \tau^{\prime}\right)$ linear und stetig. Dics gibt Anlaß zur Definition eines Funktors

$$
\because \psi^{\circ}: L V_{r} \longrightarrow K V,
$$

der definiert ist durch:

$$
\psi^{\rho}(\mathrm{E}, \tau)=\left(\mathrm{E}, \psi^{\circ} \tau\right)
$$

fur jeden Limesvektorraum $(E, \tau)$ und

$$
\psi^{O}\left(f:(E, \tau) \longrightarrow \times\left(F, \tau^{\prime}\right)\right)=f:\left(E, \psi^{\rho} \tau\right) \longrightarrow\left(F, \psi^{\prime}\right)
$$

für jeden Morphismus $f:(E, \tau)-\times\left(F, \tau^{\prime}\right)$ aus LV . (1.8) Man kann nun noch zusätzlich den Funktor der nächstgröberen vertorraumtopologie $\$$ auf LV mit Bild in TV einführen:

Für einen Limesvektorraum $(E, \tau)$ sei wr die (eindeutig 
bestimmte) Iineare (a.h.: mit der Linearstruktur von E verträgliche) ropologie auf $E$, so daß fü jeden topologischen Vektorraum $(F, \Lambda)$. und jede stetige, lineare Abbildung $f:(E, \tau) \longrightarrow x, f)$ das folgende Diagramm in $L V$ kommutiert:

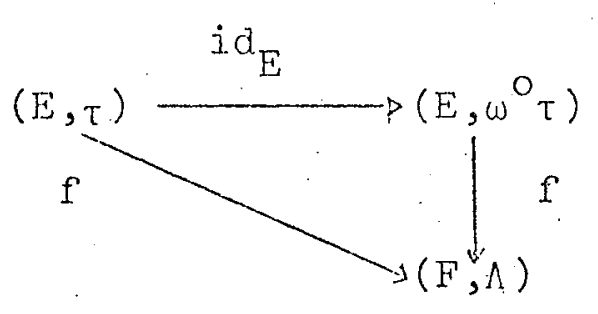

Die Topologie $\omega^{0} \tau$ wird wie folgt konstruiert:

Sei $\Omega=\{\Lambda \mid \Lambda$ lineare Topologie auf $E$, so das

$$
\left.i d_{E}:(E, \tau) \longrightarrow(E, \Lambda) \text { stetig }\right\}
$$

Es gilt stets $\Omega \neq \varnothing$, denn wie man sofort sieht, ist die indiskrete Topologie stets in $\Omega$ enthalten. Man bilde nun das topologische Produkt $\Pi(E, \Lambda)$ und betrachte die Diagonalabbildung $\Delta: E \stackrel{\Lambda \varepsilon \Omega}{>} \mathbb{R}(\mathrm{E}, \Lambda)$, die durch $\Delta(\mathrm{x})=\left(\mathrm{x}_{\Lambda}\right)_{\Lambda \varepsilon \Omega}$ mit $\mathrm{x}_{\Lambda}=\mathrm{x} \stackrel{\Lambda \varepsilon \Omega}{\mathrm{f} u ̈ r} \mathrm{x}$ aus $\mathrm{E}$ definiert ist. $\omega^{n} \mathrm{~T}$ definiere man nun als die von der linearen und injektiven Abbildung $\Delta$ und dem Produkt $\Pi(E, \Lambda)$ auf $\mathrm{E}$ induzierte Initialtopologie, aie Iinear $\mathrm{\Lambda} \varepsilon \Omega$, und rechnet nach:

i) $\omega^{\circ} \tau \quad \varepsilon \Omega$.

ii) Für $\Lambda$ aus $\Omega$ ist $i d_{E}:\left(E, \omega^{\circ} \tau\right)-(E, \Lambda)$ stetig. iii) Wenn für einen topologischen Vektorraum ( $\left.F, \Lambda^{\prime}\right)$ die lineare Abbildung $f:(E, \tau) \longrightarrow\left(F, \Lambda^{\prime}\right)$ stetig ist und wenn $\quad \Lambda_{f}$ dje (Iineare) Initialtopologie der Abbildung $f: E \longrightarrow\left(E, \Lambda^{\prime}\right)$ ist, so folgi aus der Charakterisierung einer Initialtopologie die stetigkeit von $i d_{E}:(E, \tau) \longrightarrow\left(E, \Lambda_{f}\right)$ 
und mithin $\Lambda_{f} \in \Omega$. Demnach ist $i d_{E}:\left(E, \omega^{\circ} \tau\right) \longrightarrow\left(E_{,} \Lambda_{f}\right)$ stetig, und màn erhält dje stetigkeit von

$$
f:\left(E, \omega^{O} \tau\right) \longrightarrow(F, \Lambda) \text {. }
$$

Sind nun $(E, \tau)$ und $\left(F^{\prime}, \tau^{\prime}\right)$ zwei rimésvektorräume und ist $f^{\prime}(E, T) \longrightarrow\left(F^{\prime}, T^{\prime}\right)$ sowohl stetig als auch linear, so ist auch $f:\left(E, \omega^{O} \tau\right) \longrightarrow\left(F ; \omega^{\circ} \tau^{\prime}\right)$ stetig und Iinear. Das gibt Anlaß zur Definition des Funktors der nächstgröberen linearen Topologie $\omega^{\circ}: \mathrm{LV} \longrightarrow$ TV, der bestimmt wird durch:

$$
\omega^{O}(E, \tau)=\left(E, \omega^{\circ} \tau\right)
$$

für jeden Jimesvektorraum $(E, \tau)$ und

$$
\omega^{c}\left(f^{\prime}:(E, \tau) \longrightarrow\left(F, \tau^{\prime}\right)\right)=f^{\prime}:\left(E, \omega^{0} \tau\right) \longrightarrow\left(F, \omega^{0} \tau^{\prime}\right)
$$

für jeden Morphismus $f:(E, \tau) \longrightarrow\left(F, \tau^{\prime}\right)$ aus $L V$.

Bemerkungen: Die Einschränkungen der soeben eingeführten Funktoren auf die Bilakategorien, die ja volle Unterkategorien der Definitionsbereiche der Funktoren sind, ergibt stets den identischen Funktor der Bildkategorie. Das bedeutet: Für jeden topologischen Raum (E, $\Lambda$ ) gilt stets:

$$
\ddot{\omega}(E, \Lambda)=(E, A) \text {. }
$$

Analog ergibt der Funktor $w$ angewendet auf eine stetige Abbildung eines topologischen Raumes in einen anderen topologischen Raum wieder dieselbe Abbildung mit derselben Quelle und demselben Ziel. Dasselbe gilt für den Funktor $\omega^{O}$ angewandt auf topologische vektorräume und stetige, Iineare Abbildungen zwischen topologischer Vektorräumen sowie für den Funktor $\psi^{\circ}$ angewandt auf lokalkonvexe, topologische vektorräume und stetise, lineare Abbildungen zwischen Räumen 
dieser Kategorie. Es werde noch erwähnt - was aus (1.7) und (1.8) unnittelbar ersichtich ist - das für jeden Limesvektorraum $(E, \tau)$ stets $\quad \psi^{0} \tau:=\psi^{\circ}\left(\omega^{0} \tau\right)$ gijt und $i d_{E}:\left(E, \omega^{\circ} \tau\right) \longrightarrow\left(E, \psi^{O} \tau\right)$ stetig ist.

(1.9) Inaktive Limites:

Eiń induktives System von Limesräumen werde wie folgt definiert: Sei I ein bezüglich einer ordnung nach oben gerichtetes Indexsystem. $\left(E_{i}\right)_{i \varepsilon I}$ sei ein entsprechend der Ordnung von I gerichtetes Mengensystem, d.h., für $i_{1}$ und $i_{2}$ aus $I$ mit $i_{1} \leq i_{2}$ sei $j_{i_{1} i_{2}}: E_{i_{1}} \longrightarrow E_{i_{2}}$ die Inklusionsabbildung. Für jeden Index $i$ aus $I$ sei $\tau_{i}$ eine Limitierung auf $E_{i}$ derart, daß für $i_{1} \leqq_{2}$ die Abbildung $j_{i_{1} i_{2}}:\left(E_{i_{1}}, \tau_{i_{1}}\right) \longrightarrow\left(E_{i_{2}}, \tau_{i_{2}}\right)$. stetig ist. Ferner sei $E=U_{i \varepsilon I} E_{i}$ der mengentheoretische induktive Limes des vorgegebenen induktiven systems. Für jeden Index $i$ aus $I$ sei $j_{i}: E_{i} \longrightarrow E$ : die Einbettungsabbildung. Fischer [3] hat bewiesen, daß asuf $E$ eine eindeutig bestimte Iimitierung......gibt, die man auch mit ind $\tau_{i}$ bezeichinèt, so daß für jeden Limesraum $\left(F, \tau^{\prime}\right)$ und jedes System von stetigen Abbildungen $f_{i}:\left(E_{i}, \tau_{i}\right) \longrightarrow\left(F, \tau^{\prime}\right)$, wo $i$ aus $I$ sei, das f'ur $i_{1} \leqq i_{2}$ den Bedingungen $f_{i_{1}}=f_{i_{2}} \circ j_{i_{1} i_{2}}$ genügt, genau eine stetige $A b b i l d u n g \quad f:(E, \tau) \stackrel{1}{-}(F, \tau)$ existiert, so daß das folgende Diagramm in $L$ kommutiert :

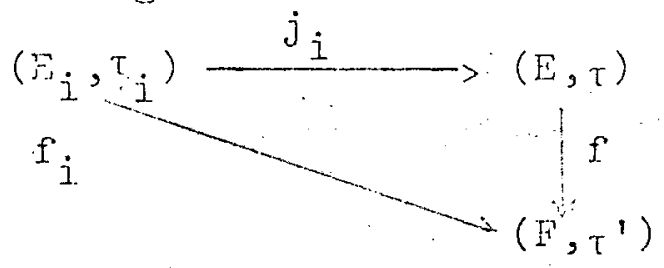


Fischer hat gozeigt: Wenn die $\left(E_{i}, T_{i}\right)$ ausätzlich simesvektorräume sind, so ist auch, wenn man $E=\mathcal{L}_{i \varepsilon I} E_{1}$ mit der in natüricher Weise definierten Vektorrauritruktur versieht, (E, ind $\tau_{i}$ ) ebenfalls ein Limesvektorraum. Die Existenz von induktiven Limites in $L$ impliziert also automatisch die Existenz von induktiven Limites in $L V$. Ferner ist (E, ind $\tau_{i}$ ) genau dann separiert, wenn jeder der Limesvektorräume $\left(E_{i}, \tau_{i}\right)$. separiert ist. Um einen induktiven Limes in der Kategorie LV zu bilden, braucht man ihn also nur in der Kategorie $L$ zu berechnen. Im Folgenden werden der in der Kategorie LV gebildete induktive Limes eines induktiven systems $\left(\left(E_{i}, \tau_{i}\right), j_{i_{1} i_{2}}\right)$ alis LV mit ind $(L V)\left(E_{i}, \tau_{i}\right)$ und die induktive Limitierung des Mengenlimes mit ind $(L V) \tau_{i}$ bezeichnet.

Sei nun $\left(\left(E_{i}, \Lambda_{i}\right), j_{i_{1} i_{2}}\right)$ ein inauktives system topologischer Yektorräume. Dann kann ind $(L V)\left(E_{i}, \hat{k}_{i}\right)=$

$=\left(\bigcup_{i \in I} E_{i}, i_{I}(L V) \Lambda_{i}\right)$ gebildet werden. Sei $\operatorname{nun}\left(F, \Lambda^{\prime}\right)$ ein topologischer Vektorraum Für j. aus I. seien linesre und stetige Abbildungen $f_{i}:\left(E_{i}, \Lambda_{i}\right) \longrightarrow\left(F, \Lambda^{\prime}\right)$ gegeben, die den Bedingunger $f_{i_{1}}=f_{i_{2}} j_{i_{1} i_{2}}$, wo $i_{1} \leqq i_{2}$ gelte, genügen. Dann gibt es eine eindeutig bestimmte Abbildung. $f:\left(E, \underset{I}{\operatorname{ind}}(L V) \Lambda_{i}\right) \longrightarrow\left(F, \Lambda^{\prime}\right)$, so daß das Diagramm

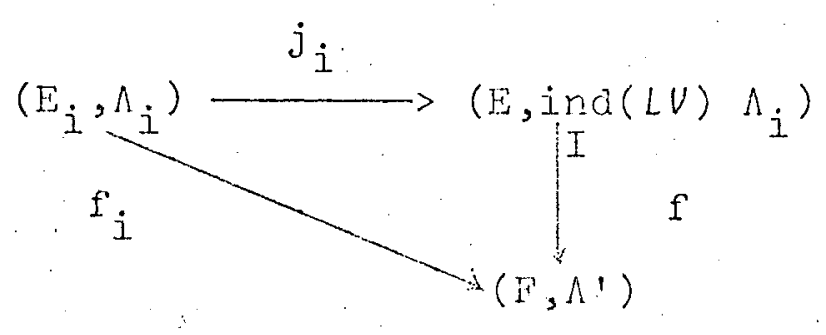

für jeden Index $i$ aus $I$ in $L V$ kommutiert. Hierbej wurde $E=\underset{i \in I}{V} E_{i}$ gesetzt. Die Anwendung des Funktors 
$\omega^{\circ}$ funrt diese kommutativen Diagrame in kommutative Diagramme

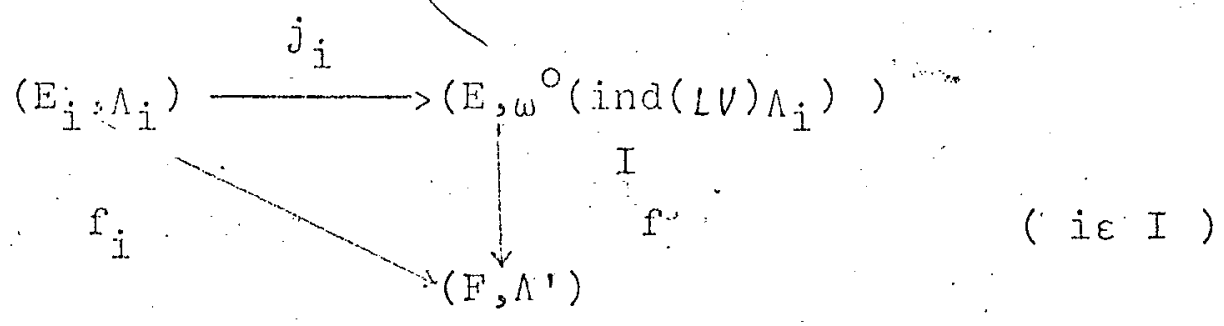

in der iatgeorie TV uber. (Man beachte, daß $\omega^{0} \Lambda_{i}=\Lambda_{i}$ und $\omega^{\circ} \Lambda^{\prime}=\Lambda^{\prime}$ nach den Voraussetzungen gilt.) Nach der algemeinen Definition des induktiven Limes folgt dann aber, daß $\left(E, \omega^{\circ}\left(\underset{I}{\operatorname{in}}(L V) \Lambda_{i}\right)\right)$ die Eigenschaften eines induktiven Limes in der Kategorie TV besitzt. Analog zeigt man: Ist $\left(\left(E_{i}, \Lambda_{i}\right), j_{j_{1} i_{2}}\right)$ ein induktives System lokalkonvexer, topologischer Vektorräume, so ist $\left(E, \psi^{O}\left(\operatorname{ind}(L V) \Lambda_{i}\right)\right)$ der induktive Limes dieses systems in der Kategorie $K V$ der lokalkonvexen, topologischen Vektorräume. Ferner gilt in der Kategorie der Limesvektorräume das folgende Diagram:

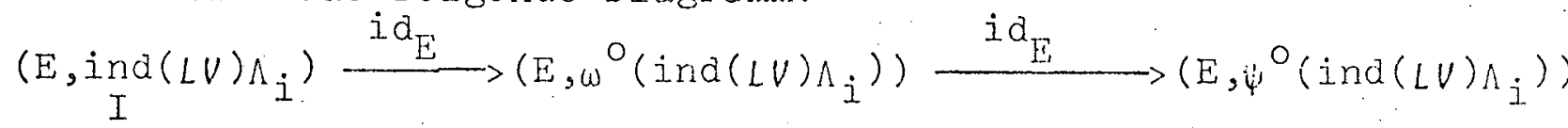

\section{Ein Lemma über schwache Topologien.}

\section{(2.1) Lemma: Seien $E$ ein Vektorraum uiber $K$, , o eine} schwache Topologie und $\Lambda$ eine lineare Topologie auf $E$ derart, dab id $_{E}:(E, \sigma) \longrightarrow(E, \Lambda)$ stetig ist. Dann ist auch $\Lambda$ eine schwache ropologie auf $E$. ( Hierbei werde unter einer schwachen ropologie auf $E$ die rnitialtopologje von E bezagrich eines Tejureuns des Dualraums von E verstanden.)

Bewejs: Sei $(E, \dot{\sigma})^{\prime}=E^{\prime}$ der Dualraum von $(E, \sigma)$. Es gilt $\sigma=\sigma\left(E, E^{\prime}\right)$, wobei $\sigma\left(E_{,} E^{\prime}\right)$ die von der Dualität (E,E') 
auf E erzeugte trwache Iopologie bedeute. Um zu zeigen, daß $\Lambda$ eine schwache Topologie.ist, genügt es zu beweisen, daß es zu jeder $\Lambda$-Nullumgebung u Funktionale $e_{1}^{1}, \ldots, e_{n}^{\prime}$ aus $(E, \Lambda)^{\prime}$ und $\hat{\delta}>0$ derart gibt, daß für $x$ aus $E$, das den Relationen $\left|<x, e_{i}\right\rangle \mid<\delta$ fur $\dot{i}=1, \ldots, n$ genügt, stets $x \in U$ folgt.

Da jeder topologische Vektorraum regulär ist, kann man zu $U$ stets eine $\Lambda$-Nullumgebung $V$ finden, so daß ihre abgeschlossene Hülle $V^{-}$( bezüglich $\Lambda$ !) die Relation. $\mathrm{V}^{-}+\mathrm{V}^{-} \subseteq U$ erfüllt. Wegen uer Stetigkeit von $\operatorname{id}_{E}:(E, \sigma) \longrightarrow(E, \Lambda)$ ist die $\Lambda$ - Nuilumgebung $V$ auch eine $\sigma$-Nullumgebung. Da $\sigma$ eine schwache Topologie auf $E$ ist, gibt es einen Unterraum $F$ von $E$, der endiche Kodimension besitzt und für den $F=V$ gilt. Insbesondere liegt dann wegen $\mathrm{V}^{-} \subseteq \mathrm{U}$ auch die abgeschlossene Hülle $F^{-}$( bezüglich $\left.\Lambda !\right)$ von $F$ in $U_{\sim}$. Es sei nun $\left(\mathrm{E} / \mathrm{F}^{-}, \Lambda_{Q}\right)$ der endlichdimensionale Quotientenraum von $E$ nach $\mathrm{F}^{-}$mit der linearen Quotiententopologie $\Lambda_{Q}$, die durch den natürlichen Homomorphismus $U:(E, \Lambda) \longrightarrow E / F^{-}$ induziert wird. $\mathrm{Da} \cdot \mathrm{F}^{-}$abgeschlossen ist, ist $\Lambda_{Q}$ separiert. $\mathrm{Da} \mathrm{E} / \mathrm{F}^{-}$endlichdimensional. ist, stimmt $\Lambda_{Q}$ nach dem Satz von Tychonoff mit der natülichen Topologie $\Lambda_{\text {nat }}$ von $\mathrm{E} / \mathrm{F}^{-}$überein. Ferner ist $v(\mathrm{U})$ Nullumgebung in $\left(E / F^{-}, \Lambda_{n a t}\right)$, denn $U(U)$ unfaBt $U\left(V^{-}\right)$, und es ist $U^{-1}\left(U\left(V^{-}\right)\right)=V^{-}+\mathrm{F}^{-}$eine Nullumgebung bezüglich $\Lambda$ : Seinun din $\mathrm{K}_{\mathrm{K}}\left(\mathrm{E} / \mathrm{F}^{-}\right)=n$. Dann gibtes, da $\mathrm{E} / \mathrm{F}^{-}$die natürliche Topologie trägt, n linear unabhängige Funktionale $f_{1}^{\prime}, . . ., f_{n}^{\prime}$ auf $E / F^{-}$und $\delta>0$ derart, das fur jedes 
Ejement $z$ aus $R / T^{*}$, das für $j=1, \ldots, n$ den Relationen $\left|<z, f_{j}^{\prime}>\right|<\delta^{-}$genügt, folgt, daß z $\varepsilon \cup\left(V^{-}\right)$gilt. Man betrachte nun die stetigen, linearen Funktionale $e_{i}^{\prime}=f_{i}^{i \cdot u}$ auf $E$. Fiur alje $x$ aus $E$, dje dje Beziehungen $|<x, e !>|<\delta$, wo $i=1, \ldots, n$, erfüllen, folgt nach Konstruktion der $f_{i}^{\prime}$, daß $U(x) \varepsilon^{\prime} U\left(V^{-}\right)$gelten muß. Also ist $x \in U^{-1}\left(U\left(V^{-}\right)\right)=V^{-}+F^{-}$. Wegen $V^{-}+F^{-} G V^{-}+V^{-} S U$ folgt also $x \in U$. Damit ist bewiesen, daß $\Lambda$ eine schwache Topologie ist.

\section{Induktive Systeme schwacher Topologien.}

Im folgenden Abschnitt soll nun das in der Einleitung erwähnte induktive system lokalkorvexer Topologien, dessen induktiver Limes sowohl in der Kategorie der topologischen Vektorräume als auch in der Kategorie der lokalkonvexen, topologischen Vektorräume die indiskrete Topologie ist, konstruiert und das gesuchte Gegenbeispiel. zum erwähnteñ Satz von Fischer angegeben werden.

(3.1)Lemma: Sei $B$ eine Menge, deren Karainalzahl gröfer als $\delta_{0}$ sei $^{\circ}\left({ }^{\circ} \mathrm{Cz}>f_{0}\right)$. Dann existiert eine Partition $\left(C_{j}\right)_{j \varepsilon} J \quad($ wo $J$ eine Indexmenge sej, ) von $B$ derart, daß füralle $j$ aus $J$ gilt $\mathrm{Cz} \mathrm{C}_{j}=\varepsilon_{0}^{\mathrm{b}}$. Das bedeutet: Die Menge B läbt sich in ein system von paarweise disjunkten Teilmengen zerlegen, die alie von abzahlbarer Mächtigkeit sind.

Beweis: Man definiere eine Klasse $\Sigma$ von Mengensystemen wie folgt:

$\Sigma$ bestehe aus alien systemen $u$ von Teilmengen von $B$, 
die folgenden Bedingungen gehorchen:

a) Jedes Element von $N$ ist von abzählbarer Mächtigkeit.

b) Je zrei verschiedene Elemente aus $N$ sind aisjunkt.

Die Klasse $\Sigma$ ist nicht leer, denn nach Voraussetzung ist $C z B>H_{0}$. Wie aus der mengenlehre bekannt ist, entnält $B$ eine abzählbare Teilmenge $B_{1}$. Dann ist aber $\left\{B_{1}\right\}$ aus $\Sigma$. Definiert man nun auf $\Sigma$ eine oronung durch die Inklusion der Elemente aus $\Sigma$, so beweist man leicht, daß $\Sigma$ induktiv georonet ist. Bezüglich dieser ordnung existieren nach dem Zonnschen Lemma maximale Elemente in $\Sigma$. Sej $M$ ein maximales Element. Es werde angenommen, daß $M$ von der Form $M=\left\{B_{j} \mid j \in J\right\}$ ist, wo $J$ eine Indexmenge sei. Dann muß $\mathrm{Cz}\left(\mathrm{B} \backslash\left(\bigcup_{j \in J} \mathrm{~B}_{j}\right)\right)<\$_{0}$ gelten. Wäre dies nämlich nicht der Fall, so ließe sich eine abzählbare Teilmeng:e $B^{\prime}$ aus $B \backslash\left(\cup_{j \in J} B_{j}\right)$ auswählen. Dann würde das System $M \cup\left\{B^{\prime}\right\}$ in $\Sigma$ liegen und $M$ umfassen, was ein Widerspruch zur, Maximelität von $M$ wäre. Folgiich muß für jedes maximale. System $\left\{\mathrm{B}_{j} \mid L_{j} \in J\right\}$ die obige Relation gelten. Mit Hilfe eires maximalen systems $M$ wird die gesuchte Partition konstruiert. Hierzu wähle man einen festen Index $j_{1}$ aus $J$ und definiere $C_{j_{1}}=B_{j} \cup\left(B \backslash\left(\underset{j \in J}{C_{j}} B_{j}\right)\right)$ sowie $C_{j}=B_{j}$ füralle $j$ aus $J$ mit $j \neq j_{1}$. Dann ist $\left(C_{j}\right)_{j \in J}$ eine Partition von $B$, die das Gewunschte leistet.

Im Folgenden sei Po der Raum aller Polynome, aufgefaßt als Funktionen auf dem Intervall $[0,1]$, mit Koeffizionten in $K$ und verschwindendem konstanten Term : 
$P_{0}=\left\{f \mid f:[0,1] \longrightarrow K, f(x)=\sum_{i=1}^{n} a_{i} x^{i}\right.$ fü $\left.x \in[0,1]\right\}$

Ferner definiere man für eine naturliche zahl $k$ :

$\begin{aligned} P_{k}=\{g \mid g:[0,1] \longrightarrow K, g(x) & =\sum_{i=2^{k}}^{m} a_{i} x^{i}, a_{i} \varepsilon K, \\ m & \left.\geq 2^{k}, x \varepsilon[0,1]\right\} .\end{aligned}$

Für jede natürliche Zahl $k$ sind $P_{k}$ und $P_{0}$ Vektorräume über $K$, die, versehen mit der Topologie der gleichmäßigen Konvergenz auf $[0,1]$, zu normierten Räumen werden. Dàbei gilt stets $\operatorname{dim}_{K} P_{k}=\operatorname{dim}_{K_{0}}=s_{0}$.

$\frac{(3.2) \text { Satz: Jedei Raum }}{\infty} P_{k}$ ist djcht in $P_{0}(k=0.1, \ldots)$. Ferner ist $\bigcap_{k=0}^{\infty} P_{k}=\{0\}$.

Beweis: Es genügt zu zeigen, daß für $k=0,1, \ldots$ der Raum $\mathrm{P}_{\mathrm{k}+1}$ dicht in $\mathrm{P}_{\mathrm{k}}$ ist. Sej $f$ aus $\mathrm{P}_{k}$ von der Form $f(x)=\sum_{i=2}^{m} k a_{i} x^{i} \quad(x \in[0,1])$.

Ohne Beschränkung der Allgemeinheit werde angenommen, daß $m \geq 2^{k+1}$ gilt. Nach den Approximationssatz von Weierstrass existiert eine Folge $\left(g_{n}\right)$ von Polynomen auf $[0,1]$, die die Tunktion $\quad x \longmapsto x^{1 / 2} \quad$ gleichmäßig approximiert. Hierbei kann ohne Einschränkung der Allgemeinheit angenommen werden, daß die Folge $\left(g_{n}\right)$ in $P_{0}$ liegt. Definiert man für natürliche Zahlen $n$ und $i$ das Polynom $E_{n i}$ als Funktion auf $[0,1]$ durch $g_{n j}(x)=g_{n}\left(x^{2 i}\right)$, so impliziert dies, das die Folge (Eni) neN auf $[0,1]$ gleichmäßig die Funktion $x \longmapsto x^{j}$ approximiert. Da für alle $\mathrm{g}_{\mathrm{n}}$ die konstanten Koef'izienten verschwinden, folgt, daß für die $\mathrm{g}_{\mathrm{rij}}$ die Koeffizienten aller Terme vom Grade $j<2 j$ verschwinden müssen. Insbesondere folgt fur jede 
natürliche zant $k$ und $i \geq 2^{k}$ : Die Polynome Sni $_{n}$ liegen alle in $P_{K+1}$ und approximieren gleichmäßj.g die Funktion $x \longrightarrow x^{j}$. Man definiere fur jede natürliche Zahl $n$ nun ejn polynom $f_{n}$ aus $\mathrm{P}_{k+1}$ durch

$$
f_{n}(x)=\sum_{i=2^{k}}^{2^{k+1} i} a_{i} g_{n j}(x)+\sum_{i=2}^{m} k+1 a_{i} x^{i}(x \varepsilon[0,1]) \text {. }
$$

Man erkennt nun, daß die Folge $\left(f_{n}\right)$ gleichmäßig das Polynom $f$ auf $[0,1]$ approximiert. Infolgedessen ist $\mathrm{P}_{\mathrm{k}+1}$ dicht in $\mathrm{P}_{\mathrm{k}}$ und somit die erste Behauptung des Satzes bewiesen. Die zweite Behauptung zu verifizieren ist trivial.

(3.3) Definition: Für einen K-Vektorraum $E$ sej. $E^{*}$ der Raum aller K-linearen Funktionale auf $E$. Ein Unterraum $M$ von $E^{*}$ heise total, wenn aus den Relationen $x \in E$ und $\langle x, m>=0$ fur alie $m$ aus $M$ folgt: $x=0$. (̈̈quivalent zur Totalität des Raumes M ist die Separiertheit der von $M$ auf $E$ induzierten schwachen Topologie $\sigma(E, M)$.

Auf $P_{0} \times P_{0}$ werde ein bilineares Funktional $b(\ldots)$ definiert durch:

$$
b(f, g)=\int_{0}^{1} f(x) g(x) d x \quad\left((f, g) \varepsilon P_{0} \times P_{0}\right) .
$$

Dann ist die Abbildung $\quad \mathrm{I}: \mathrm{P}_{\mathrm{O}} \longrightarrow \mathrm{P}_{\mathrm{O}}^{\dagger}$ definiert durch $I(g)=b(\cdot, g) \quad\left(g \in P_{0}\right)$, wie man sich sofort uberlegt, eine Einbettung von $P_{0}$ in $P_{0}^{*}$, die die fólgenden Eigerschaften besitzt:

a) Sie ist linear und injektiv.

b) Jeder bezüglich der Supremumsrorm auf $[0,1]$ dichte Unterraum $F$ von $P_{0}$ wird auf einen totalen Unterraum von $p_{0}^{*}$ abgebildet; denn ist $f$ aus $p_{0}$, so auch $\bar{f}$. 
( Hierbei sei $\vec{f}=f$, falls $\mathrm{K}$ der Körper der rellen Zahlen ist. Falls $K$ der Körper der komplexen Zahlen ist, sei $\bar{f}$ die zu $\mathrm{f}$ konjugjert komplexe Funktion.) Dann aber gibt es ejne Folge $\left(h_{n}\right)$ aus F, die gleichmäßig gegen $\bar{f}$ konvergiert. Daraus folgt aber, daß

$$
b\left(f, h_{n}\right)=\int_{0}^{1} f(x) h_{n}(x) d x
$$

gegen

$$
b(f, \bar{f})=\int_{0}^{1}|f(x)|^{2} d x
$$

konvergiert. Nun gilt $b(f, \bar{f})=0$ genau dann, wenn $f=0$ gilt. Aus $f \in P_{0}$ und $b(f, h)=0$ für alle $h$ aus $F$ folgt somit wegen der Dichte von $F$ in $P_{0}$; daß $f=0$ gelten muß, daß also $I(F)$ total ist. Es werden also die Räume $P_{k}$ durch die Abbildung $I$ auf totale Unterräume $P_{k}^{\prime}=I\left(P_{k}\right)$ von ${ }_{0}$ abgebildet. Wegen der Injektivität von I gilt:

$$
P_{k+1} \leq P_{k}^{i} \quad \text { und } \bigcap_{k=1}^{\infty} P_{k}^{k}=\{0\} \text {. }
$$

Das Ergebnis dieser Betrachtung kann zu folgendem Korollar zusammengefaßt werden:

(3.4) Korollar: Es existiert eine Folge ( $\left.\mathrm{P}_{\mathrm{k}}^{\prime}\right)$ totaler Unterräume von $P_{0}$ derart, daß gilt:

a) $\mathrm{P}_{\mathrm{k}+1}^{\prime} \subseteq \mathrm{P}_{\mathrm{k}}^{\prime}$.

b) $\bigcap_{k=1}^{\infty} \cdot P_{k}^{i}=\{0\}$.

Ist nun $E$ ein beliebiger $k$-Vektorraum mit $\quad \operatorname{dim}_{K} E=$ sh $_{O^{3}}$ so existiert wegen $\operatorname{dim}_{K} P_{O}=\$ \$_{0}$ ein $K$-Isomorphismus Is $: E \longrightarrow P_{0}$. Die adjungierte Abbildung (Is $)^{*}: \mathrm{P}_{0}^{*} \longrightarrow \mathrm{E}^{*}$ ist dann ebenfalis ein Isomorphismus. Durch $(I s)^{*}$ werden totale Unterräume auf totale Unter- 
räume abgebildet. Da (Is)* bijektiv ist, eriält man, wern man $E_{k}=\left(I_{s}\right) *\left(P_{k}\right)$ setzt, $(k=0,1, \ldots,)_{*}:$ (3.5) Korol1ar: Zu jedem K-Vektorraum a mit dim $\mathrm{K}=$ st existient ejne Folge $\left(\mathrm{E}_{\mathrm{K}}^{\prime}\right)$ totaler Unierräume von $E$. mit den folgenden Eigenschaften:
a)
$E_{k+1}^{\prime}=E_{k}^{\prime}$
$(k=0,1, \ldots)$. .
b)
$\mathrm{N}_{\mathrm{k}=1}^{\mathrm{i}}=$
$\{0\}$

Dieses Ergebnis soll nun' auf unendichdimensionale $K$ - Vektorräume beliebiger Dimension übertragen weraen:

(3.6) Satz: Sei E ein beliebiger K-Vektorraum mit $\operatorname{dim}_{K} E \geqq s_{0}$. Dann existjert eine Folge $\left(E_{K}^{\prime}\right)$ totaler Unterräume von $E^{*}$, so daß die folgenden Bedingungen erfülit sind:
a) $E_{k+1}^{\prime} \subseteq E_{k}^{\prime}$
$(k=0,1, \ldots)$.
b)
$\bigcap_{k=1}^{\infty} E_{k}^{\prime}=\{0\}$.

Beweis: Fü den Fali dim $\mathrm{K}=$ s stinmt die Aussage (3.6) mit der von (3.5) überein. Es genügt also, den Fail $\operatorname{dim}_{K} E \geqslant{ }^{3}, z u$ betrachten. Sei $B$ eine Basis von $E$. Dann ist $C z B=\operatorname{dim}_{K} E$. Fernersei $\left(B_{j}\right)_{j \varepsilon} J$ eine Partition dieser Basis in abrählbare Tejlmengen, die nach (3.1) existiert. Es sei für jeden Index $j$ aus $J$ stets $E_{j}$ der von $B_{j}$ in $E$ aufgespannte Unterraum. Es gilt dim $\mathrm{E}_{j}=\varsigma_{0}$. Dann läßt sich $\mathrm{E}$ wie folgt als direkte Summe der $E_{j}$ darstellen:

$$
E=\underset{j \varepsilon J}{\oplus E_{j}}
$$

Nach (3.5) existiert fir $E_{j}$ eine Folge (E!,n)n EN totaler Unterräume von $\mathrm{E}_{j}^{*}$, die den Bedingungen (a) und 
(b) Von (3.5) geniigt.

Nach bekannten. Satzen aus der jjoearen Algebra jabt sich dex Dualraum $\mathrm{E}^{*}$. von $\mathrm{E}$ als airektes Produkt der Dualräume $E_{j}^{*}$ von $E_{j}$ därstelien:

$\mathrm{E}^{*}=\underset{j \varepsilon j}{\Pi} \mathrm{E}_{j}^{*} \cdot$

Für $k=0,1, \ldots$ definiere man nun Unterräume $E_{k}^{\prime}$ von $\mathrm{E}^{*}$ durch :

$$
E_{k}^{\prime}=\prod_{j \in J} \cdot E_{j, k} \cdot
$$

Da nach Wahl der Räume $E_{j, k}^{j}$ jeder dieser Räume total in $E_{j}^{*}$ ist, folgt, daß $E_{k}$ für $k=i, 1, \ldots$ total $\dot{i n}_{n}$ $E^{*}$ sein muß. Aus der Konstruktion der Rämme $E_{k}^{\prime}$ folgt nun unmittelbar, daB für $k=0,1, \ldots$ gelten muß:

$$
E_{k+1}^{\prime} \leq E_{k}^{\prime}
$$

Ferner folgt aus $\bigcap_{k=1}^{\infty} E_{j, k}=\{0\}$ für jeden Index $j$ aus $J$ nach Konstruktion der Räume $E_{k}^{\prime}$ :

$$
\bigcap_{k=1}^{\infty} E_{k}^{\prime}=\{0\}
$$

Aufgrund dieses Satzes ist nun jedem unendichdimensionalen K-Vektorraum $\dot{E}$ " eine Folge $\left(E_{k}^{\prime}\right)$ totaler Unterräume von $E^{*}$ so zugeoranet, daß die Bedingungen (a) und (b) in (3.6) erfültt sind.

Jeder der Dualitäten $\left(E, E_{k}^{\prime}\right) \quad(k=0,1, \ldots)$ entspricht nun in eindeutiger heise eine schwache Topologie $\dot{\sigma}_{k_{2}}=\sigma\left(E, E_{k}^{r}\right)$; die vegen der Totalität von $E_{k}^{3}$ in $E^{*}$ separiert ist. Für natürliche Zahlen $k$ und $k^{\prime}$ mit $k \leq k^{\prime}$ gilt $E_{k^{\prime}} \leq E_{k}^{\prime}$. Aufgrurd der Eigenschaft einer schwachen Topologie, Initialtopologie zu sein, folgt 
die stetigkeit der Abbilang $i a_{E}:\left(E, \sigma_{k}\right) \longrightarrow\left(E, \sigma_{k}\right)$. Damit ist ejrinduktives system $\left(\left(\mathrm{E}, \sigma_{k}\right)\right.$, id $\left.\mathrm{d}_{\mathrm{E}}\right)$ definiert. Diesqs system ist abzalbar. Seinun $\left(E, \tau_{E}\right)=\operatorname{ind}(L V)\left(E, \sigma_{k}\right)$.

Nach der Eemerkung in (1.9) ist $\left(E, \tau_{E}\right)$ ein separierter Limestvektorraum (Marinescu-Raum), von dem nun gezeigt werden soll, daßsowohl $\omega^{O}{ }^{\top} \mathrm{E}$ als auch $\psi^{\circ}{ }^{\circ} \mathrm{E}$ die indiskre-te Topologie auf E sind.

Für jede natürliche Zahl $k$ ist die Abbildung

$$
i d_{E}:\left(E, \sigma_{k}\right) \longrightarrow\left(E, \omega^{\circ} \tau_{E}\right)
$$

stetig. Da die Vektorraumtopologien $\sigma_{k}$ schwache. Topologien sind, folgt nach (2.i), daß auch $\omega^{\mathrm{C}} \tau_{\mathrm{E}}$ eine schwache Topologie und somit lokalkonvex sein muß. Hieraus folgt nach (1.9), daß $\omega^{0} \tau_{E}=\psi^{0} \tau_{E}$ gelten muß. Es bleibt also zu zeigen, daß $\psi^{\circ} \tau_{E}$ die indiskrete Topologie auf $E$ ist. Hierbei werde berücksichtigt, daß nach soeben durchgeführtem Schlus $\psi^{\circ} \tau_{\mathrm{E}}$ eine schwache Topologie ist. Um die Behauptung zu beweisen, goriugt es zu zeigen, daß das Nullfunktional o das einzige bezuglich $\psi^{\circ} \tau_{E}$ stetige, lineare Funktional ist.

Wegen der Stetigkeit von $i d_{E}:\left(E, \sigma_{k}\right) \longrightarrow\left(E, \psi^{O} \tau_{E}\right)$ für jede natürliche Zahl $k$ (siehe. (1.9) ) folgt. wegen $E_{K}^{\prime}=\left(E, \sigma_{k}\right)$, für jedes stetige, lineare Funktional e' aus $\left(E, \psi^{\circ} \tau_{E}\right)^{\prime}$ auch $e^{\prime} \varepsilon E_{k}^{\prime}$. Also gilt $e^{\prime} \varepsilon \bigcap_{k=1} E_{k}^{\prime}=\{0\}$ und folglich $e^{\prime}=0$. Aus den soeben argesteliten Betrachtungen ergibt sich dann das folgende Ergebnis:

(3.7) Satz: Sei E ejn unendijchdjmensjonaler K-Vertorraum. 
Dann exjstiert auf $E$ eine seperjerte, mit der Linearstruktur von $E$ kompatible Jimitierung $\tau_{\mathrm{E}}$ derart, daB die nächstgröbere mit der vektorraumstruktur von E verträgIiche ropologie von E aie indiskrete Topologie ist.

\section{Gegenbeispiel zu ejnem Satz von Pischer.}

Im Folgenden sei $E$ ein unendichaimensionaler K-Vektorraum. $\tau_{E}$ sei eine nach dem im Abschnitt 3 angegebenen Konstruk.. tionsverfahren gewomene separierte Vektorraumlimitiering auf $E$. Betrachtet man $E$ nur mit der additiven struktur als einziger algebraischer Struktur, so ist $\left(E, \tau_{E}\right)$ eine limitierte Gruppe. Es soll nun beriesen werden:

(4.1) Satz: $\left(E, w \tau_{E}\right)$ ist keine topologische Gruope, da die fadition nicht bezüglich $\omega_{\mathrm{E}}$ stetig ist.

Beveis: Hierzu wird das folgende Lemma benötigt:

(4.2) Lemma: Sei $(E, \tau)$ ein Limesvektorraum. Die Addition auf $E$ sei bezüglich wt stetig. Dann ist die abgeschlossene Hülle $F^{-}$eines Unterraumes $F$ von $E$ bezüglich der Topologie wt wieder ein Unterraum von $E$. Beweis: Sei $\lambda$ aus $K \cdot x$ sei aus $F^{-}$. Für $\lambda=0$ folgt $\lambda \cdot \mathrm{x} \in \mathrm{F} \subseteq \mathrm{F}^{-}$. Für $\lambda \neq 0$ ist die Homothetje $j_{\lambda}: x \longmapsto \lambda \cdot x$ auf Grund der Stetigkeit der Skalarmultiplikation ein Homöomorphismus bezüglich $\tau$. Nach (1.6) folgt, daß $j_{\lambda}$ auch bezüglich $\omega \tau$ ein Homöomorphismus von $\mathrm{E}$ auf sich selbst sein muß. Da $F$ ein Iinearer Raum ist, gilt $j_{\lambda}(F)=$. Dann folgt wegen der. Homöomorphieeigenschaf't von $j_{\lambda}$ : 


$$
\lambda \cdot \mathrm{F}^{-}=j_{\lambda}\left(\mathrm{F}^{-}\right)=j_{\lambda}\left(\mathrm{F}^{-}\right)^{-}=\lambda \cdot \mathrm{F}^{-} \cdot
$$

Demach gilt fur $x$ aus $F^{-}$stets $\lambda \cdot x \in F^{-} \cdot x_{*}$ Insbesondere jst die Abbilaung $j_{-1}: x_{-}^{\longrightarrow}-x \quad(x \in E)$ bezüglich wt stetig. Interpretiert man nun $E$ als abelsche Gruppe, so ist. (E,ut) nach der Voraussetzung über die Stetigkeit der Addition bezüglich wT eine topologische, abelsche Gruppe. Da $F$ als Iinearer Unterraum von $E$ eine Untergruppe ist, ist - wie aus der theorie der topologischen Gruppen bekannt - die abgeschlossene Hülle $\mathrm{F}^{-}$von $\mathrm{F}$ bezüglich w $\mathrm{t}$ wieder eine untergruppe von E. Für $x$ und $y$ aus $F^{-}$gilt also stets $x+y \varepsilon F^{-}$. Aus diesen Betrachtungen folgt aber, daB $\mathrm{F}^{-}$ein linearer Unterraum von $\mathrm{E}$ ist.

Es werde nun (4.1) bewiesen. Hierzu werde angenommen, daf die Addition auf $E$ bezüglich ${ }^{\omega} \tau_{E}$ stetig sei, und diese Annahme zu einem Widerspruch gefürt. Da $\left(E, \tau_{E}\right)$ separiert ist, genügt $\omega \tau_{E}($ siehe $(1.5)$ ) dem ersten Trennungiaxiom. Da die Adition auf $E$ mit ${ }^{\omega} \tau_{E}$ nach Voraussetzung kompatibel sein.solite, folgt aus der Beweisfürung zu (4.2), daß $\left(E, \omega \tau_{E}\right)$ eine topologische Gruppe ist. Nach bekannten Sätzen aus der Theorie der topologischen Gruppen ist aann aber $\left(E, \omega \tau_{E}\right)$ separiert.

Sej nun $k$ eine beliebige naturliche zahl. Nach der Konstruktion von .. $\tau_{\mathrm{E}}$ im Abschnitt 3 und nach (1.6) sowie $(1.9)$ ist $\operatorname{id}_{E}:\left(E, \sigma_{k}\right) \longrightarrow\left(E, \omega \tau_{E}\right)$ stetig. Da jede topologische Gruppe regulär ist, kann man nur eine 
${ }^{\omega} \tau_{E}$ - Nuldumgebung $\mathrm{u}$ wählen, so daß die abgeschlossene Hülle $U^{-}$von $U$ bezüglich ${ }^{-} \mathrm{E}$ die Bedingung $U^{-} \neq E$ erfültt. Dann ist $U$ auch Nullumgebung bezüglich jeder der Topologien $\sigma_{k}$, wo $k$ eine beliebige natürliche Zahl. sei. Demach mu U einen Unterraum $F$ von endicher Kodimension enthalten. Nach (4.2) ist die abgeschlossene Hülle $F^{-}$von $F$ bezüglich $\omega_{E}$ ein echter linearer Unterraum von $E$. Wiederum wegen der stetigkeit von $\operatorname{id}_{E}:\left(E, \sigma_{k}\right) \longrightarrow\left(E, \omega \tau_{E}\right)$ für jede natürliche Zahl $k$ folgt; daß $\mathrm{F}^{-}$auch bezügljch jeder der Topologien $\sigma_{\mathrm{k}}$ abgeschlossen ist. Dann ist für $k$ aus $N$ der Quotientenraum $\left(E / F^{-},\left(\sigma_{k}\right)_{Q}\right)$ ein endichdimensionaler, separierter topologischer Vektorraum . Da nach dem Satz von Tychonoff auf $E / F^{-}$aber nur eine separierte, kompatible Topologie $\Lambda_{\text {nat }}$ existieren kann, foigt fur jedes $k$, daß $\left(\sigma_{k}\right)_{Q}=\Lambda_{\text {nat }}$ gilt. Bezoichnet man den natürliclen Homomorphismus $E \longrightarrow E / F^{-}$mit $U$, so folgt für jede natüriche Zahl $k$ die Stetigkeit der Abbildung

$$
U:\left(E, \sigma_{k}\right) \longrightarrow\left(E / F^{-}, \Lambda_{n a t}\right) \text {. }
$$

$\mathrm{Da}\left(\mathrm{E} / \mathrm{F}^{-}, \Lambda_{\text {nat }}\right)^{\prime}$ ein topologischer Vektorraum ist, folgt nach der Charakterisierung des induktiven Limes in (1.9) die Stetigkeit der Abbilaung

$$
u:\left(E, \omega^{0} \tau_{E}\right)=\omega^{0}\left(\underset{N}{\operatorname{ind}}(L V)\left(E, \sigma_{k}\right)\right)
$$
$\rightarrow\left(E / F^{-}, \Lambda_{n a t}\right)$ Wegen der Endlichdimensionalität von $\mathrm{E} / \mathrm{F}^{-}$und wegen $\mathrm{E} / \mathrm{F}^{-} \neq$o\} folgt, dab mindestens ein nichtverschwindendes, stetiges, lineares Funktional $\because$ auf $\left(E / F^{-}, \Lambda_{\text {nat }}\right)$ existiert. Da $u$ surjektiv und stetig ist, ist dam aber fov ein nichtverschrindendes, stetiges, ineares Furktional. 
auf ind $(L V)\left(E, \sigma_{k}\right)$. Da dieser Raum aber, wie jim Abschnitt 3 gezeigt worden war, keine stetigen linearen Furktionaje, die nicht identisch VulI sind, besitzt, ergibt sich der gewüschte viderspruch.

Berücksichtigt man, daß für jeden endlichdimensionalen, separierten Limesvektorraum $(E, \tau)$ die Limitjerung $\dot{\tau}$ schon eine Topologie ist, a.h., dab $\omega \tau=\tau$ gilt ( $\operatorname{siehe~[8]),~}$ so erhält man zusammen mit (4.1) die folgende Charakterisierung von endichdimensionalen vektorräumen über $K$ : (4.3) Der K-Vektopraum $E$ ist genau dann endlichdimensional. wenn für jede separierte, kompatible Limitierung $\tau$ auf $E$ der Raum (E,w $)$ ein topologischer Vertorraum ist. Mit (3.7) erhält man:

(4.4) Der $\mathrm{K}$-Vektorraum $\mathrm{E}$ ist genau dann endlichdimensional, wenn für jede separierte, kompatible Limitierung $\tau$. auf E die Topologien $\omega^{\circ} \tau$ und $\psi^{\circ} \tau$ separiert sind.

\section{Lokalkompakte Limesvektorräume.}

Sei $E$ eine Menge, $E \neq \varnothing$. B sei eine Filterbasis in $E$. Dann werde der von $B$ erzeugte Filter mit [B] bezeichnet. Seien $E$ und $F$ nichtieere Mengen, $f: E \longrightarrow F$ eine Abbildung, $\theta$ ein Filter auf E. Dann sei der Bildfilter $f(\theta)$ von $\theta$ unter $f$ auf $F$ definiert durch:

$$
f^{\prime}(\theta)=\left[\begin{array}{llllll}
\{f & f(T) & T & \varepsilon & \theta
\end{array}\right] .
$$

Lokalkompakte, topologische Räume lassen sich durch ihre Algebren stetiger, reellwertiger Funktionen wie folgt 
charakterisienen: Ein topologischer Raum $(E, \Lambda)$ ist genau damn lokalkompakt, wenn dje R-Algebra $C((E, \Lambda), R)$ allep stetigen Punkionen auf $(E, A)$ : mit Werten in $R$ eine gröbste Topologie " $\Lambda_{c o}$ besitzt, so daß die Evajuationsebbilaung $\quad \omega:\left(C((E, \Lambda), P), \Lambda_{C O}\right) \times(E, \Lambda) \longrightarrow R$ stetig ist. Diese Topologje ist die Topologie der gleichmäßigen Konvergenz auf kompakten Tejimengen von $(E, h)$. Ein Limesraum (E, $\tau$ ) heiße kompakt ( siehe Fischer [3]), wenn jeder Ultrafilter auf $E$ bezüglich $\tau$ konvergiert. Eine Teifmenge $M$ eines Limesraums (E, $\tau$ ) heiße kompakt, wenn. $M$ versehen mit der Relativimitierung. $\tau_{\mathbb{M}}$ bezüglich $\mathrm{t}$ kompakt ist.

Bej Untersuchungen über die stetige Konvergenz auf den AIgebren stetigor reellwertiger Funktionen, die auf Limesräumen definiert sind (siehe Binz [1] ) entdeckte Schroder, daß es auch Limesräume geben kann, die keine topologischen Räume sind, so daß die stetige Kcinergenz auf aer Algebra. der stetigen, reellwertigen Funktionen dieser Limesräume eine Topologie ist. Dies veranlaßte Schroder zur Definition des lokalkompakten Limesraumes, die nun angegeben werde: (5.1) Definition: Ein Iimesraum (E, $\tau$ ) heibe lokalkompakt, wenn jeder in (E, $\tau)$ konvergente iliter eine kompakte Menge. enthält.

Wie man sofort erkennt, stimmt diese Definition im Falle eines topologischen Raumes mit der ublichen Definition eines lokalkompakten, topologischen Raunes uberein.

In der Kategorje der Jimesräume lrasen sich nun die folfenden beiden Sitze beweisen, die in der Kategorie der topologischen 
Rüume nicht gel.ten:

(5.2) Satzi Sej $\left(\left(E_{j}, \tau_{j}\right)\right)_{j \in I}$ ( I einenicht]eere Indexmenge ) ejne Familie voi lokalkompakten Jimesraumen. E sei eine Menge mit $E \neq \dot{D}$. Ferner sei zu jedem Index. i aus I ejne Abbilaung $f_{i}: E_{i} \longrightarrow E$ gegeben, so das gilt: $\quad U_{i \varepsilon I} f_{i}\left(E_{i}\right)=E$ Die Finalimitierung, die von den Limesräumen. $\left(E_{i}, \tau_{i}\right)$ und den Abbildungen $f_{i}$ auf $E$ induziert wira, werde mit $\tau$ bezejchnet.

Behauptung: $(E, \tau)$ ist jokalkompakt.

Beweis: Sei $y$ aus $E$. Ein Filter $\theta$ auf $E$ konvergiert : bezüglich $\tau$ gegen $y$ (siehe Fischer [3]) , wenn es Indizes $i_{1}, \ldots, i_{n}$ aus $I$, Elemente $x_{j_{k}}$ aus $E_{i_{k}}$ ( wo $\left.k=1, \ldots, n\right)$ und Filter $\Phi_{i_{k}}$ aus $\tau_{i_{k}}\left(x_{i_{k}}\right)$ derart gibt, daß gilt:

$$
f_{i_{k}}\left(x_{i_{k}}\right)=y \text { und } \bigcap_{k=1}^{n} f_{i_{k}}\left(\Phi_{i_{k}}\right) E \theta \text {. }
$$

$\tau$ ist charakterisiert als die feinste luimitierung auf

E mit der Eigenschaft, daß die Abbildungen $f_{i}$; wo $i$ als

I beliebig sei, stetig sind.

Dá jeder Raum $\left(E_{i}, \tau_{i}\right)$ lokalkompakt ist, gibt es kompakte Teilmengen $\mathrm{K}_{i_{k}}$ von $\left(\mathrm{E}_{\mathrm{i}_{\mathrm{k}}}, \tau_{\mathrm{i}_{k}}\right)$ mit $\mathrm{K}_{\mathrm{i}_{k}} \varepsilon \Phi_{i_{k}}$. Nun ist

$$
\sum_{k=1}^{n} f_{i_{k}}\left(\Phi_{i_{k}}\right)=\left\{T_{1} \cup \ldots \mathrm{T}_{n} \mid \mathrm{T}_{k} \varepsilon f_{j_{k}}\left(\Phi_{i_{k}}\right)\right\} \subseteq \theta .
$$

Wegen der stetigkeit der Abbizdungen $f_{i}$ sind die Mengen $f_{i_{k}}\left(\mathrm{~K}_{i_{k}}\right)$ kompakte Teilmengen von $(E, \tau)$. Da die Vereinigung vor endich vielen kompakten Mengen wieder kompakt ist, ist $\sum_{k=1}^{n} f_{i_{k}}\left(k_{i_{k}}\right)$ eine komakte Teilmenge von $(E, \tau)$, 
die im Fijter $\theta$ entralten ist. Da 0 ein beliebiger konvergenter Filter aus $(\mathrm{E}, \mathrm{T})$ war, folgt, daß.... $\mathrm{E}, \tau)$ lokalkompakt sein mus.

Betrachtet man induktive systeme lokalkompakter limesräume ( definiert nach (1.9) ), so ergibt sich hjeraus unmittelbar: (5.3) Koroljar: Der induktive Limes von lokalkompakten Limesräumen ist lokalkompakt.

Weiter erhät man allgemein:

(5.4) Satz: Jeder lokalkompakte Limesralm läßst sich als induktiver Limes seiner kompakter Teilmengen, versehen mit der Relativlimitierung, darstellen.

Beweis: Sei $(E, \tau)$ ein lokalkompakter Jimesraum. Definiere: $K=\{K \mid K \subseteq E, K$ kompakte Teilmenge $\operatorname{von}(E, \tau)\}$. Da die endliche Vereinigung von kompa'ten Mengen wiederum kompakt ist und da jeder Punkt des Limesraumes (E, $\tau$ ) kompakt ist, folgt, daß $K$ ein bezüglich der Inklusion gerichtetes Überdeckungssystem von $E$ ist. Folglich kann man $k$ zusammen mit den in natürlicher Weise gegebenen Inklusionsabbildungen als..ein induktives system kompakter Iimesräume betrachten. Der mengentheoretische induktive Jimes dieses Systems ist $\mathrm{E}$. Für $\mathrm{K}$ aus $K$ sei ${ }^{\tau} \mathrm{K}$ die von ' $\tau$ aus $\mathrm{K}$ induzierte Relativlimitielung; $j_{\mathrm{K}}:\left(\mathrm{K}, \tau_{\mathrm{K}}\right) \longrightarrow(\mathrm{E}, \tau)$ sei die Ejnbettungsabbildung. Bezeichret man die Limitierung auf $E$, die durch das induktive System als ljmitierung des induktiven Limes definiert ist, mit. $\tau^{\prime}$, so folgt unmitelbar die stetigheit der Abbildung

$$
\text { j. } \mathrm{d}_{\mathrm{E}:}:\left(\mathrm{E}, \tau^{\prime}\right) \longrightarrow(\mathrm{E}, \tau) \quad
$$

Sei nun $x$ aus $E \cdot \odot$ sej aus $\tau(x)$. Da (E, $T$ ) 
lokalkompakt war, gibt es eine kompakte nenge $K$ in (E, $\tau$ ) , die $x$ entbilt und die in $\theta$ enthalten ist. Es, sei $\Theta_{K}$ die spur von $\theta$ auf $K$. Nach Definition der Relativijmitierung konvergiert $\Theta_{K}$ in $\left(K, \tau_{K}\right)$ gegen $x$. Da $\left(K, \tau_{K}\right)$ ein Element des betrachteten induktiven systems ist, folgt nach Definition des induktiven Limes, daß der Filter. $j_{K}\left(\theta_{K}\right)=\left[\theta_{K}=\theta\right.$ in $\left(E, \tau^{\prime}\right)$ gegen $x$ konvergiert. $\mathrm{Da} x$ aus $E$ und $\Theta$ aus $\tau(x)$ beliebig waren, ist damit die Stetigkeit von $i d_{E}:(E, \tau) \longrightarrow\left(E, \tau^{\prime}\right)$ bewiesen. Zusammen mit dem zu Beginn Geztigten folgt nun $\tau=\tau^{*}$. Sei nun $(E, \tau)$ ejn beliebiger Limesvektorraum über dem Körper $K$. Ferner sei $F$ ein endichdimensionaler Unterraum von $E \cdot T_{F}$ sei die Relatjvlimitierung, die von $\tau$ auf $F$ induziert wira. Wie zus [8] hervorgent, ist $\tau_{F}$ stets gröber als die natürliche Topologie $\Lambda_{\text {nat }}$, die $F$ als endlichdimensionalor Vektorraum trägt. Folglich ist die Einbettungsabbildung $j_{F}:\left(F, \Lambda_{n a t}\right) \longrightarrow(E, \tau)$ stetig.

Sei nun $F$ das System aller endiichdimensionalen unterräume von $E$. Zusammen mit den Inklusionsabbildungen ist dieses System ejn induktives system von Vektorräumen, dessen jnduktiver Limes in der Kategorie der $K$-Vektorräume $E$ ist. Man versehe nun jeden Vektorraum $F$ aus $F$ mit der naturi.chen Topologie. Zusammen mit den Inklusionsabbilaungen erhält man so ein induktives System von lokalkonvexen, topologischen Vektorräumen, dessen induktiver Iimes in der Kategorie der Jjmesvektorräume ( $\left.\mathrm{E}_{0} \mathrm{r}_{0}\right)$ ist, wobei $\tau_{0}$ die durch das induktive syatem auf $\mathrm{E}$ erzeugte vektorramimi- 
tierung ist. (E, $\left.\tau_{0}\right)$ besitzt die folgende charakteristische Eigenschatt :

Tiur jeden Limesvektorraum $\left(E^{\prime}, \tau^{\prime}\right)$, und fur jede lineare Abbilaung $f: E \longrightarrow E^{\prime}$ ist $f:\left(E, \tau_{0}\right) \longrightarrow\left(E^{\prime}, \tau^{\prime}\right)$ stetig. Das bedeutet: $\tau_{0}$ ist die reinste vektorraumimitierung, die $\mathrm{E}$ tragen kann. (Als induktivep Limes von lokalkonvexen, topologischen Vektorräumen ist $\left(\mathbb{E}, \tau_{0}\right)$ ein Marinescu-Raum.) Da es nun gleichgijtig ist, ob der induktive Limes in der Kategorie der Limesräume oder in der Kategorie der Limesvektorräume berechnet wird ( siehe (1.9) ) und da jeder endijchaimensionale Vektorraum, versehen mit der naturlichen Topologie, lokalkompakt ist, folgt nach (5.3), daß (E, to $)$ lokalkompakt sein muß. Man erhält also: (5.5) Satz: Sei $E$ ein beliebiger K-Vektorraum. To sei die feinste Vektorraumlinitierung, dje E besitzt. Dann ist $\left(\mathrm{E}, \mathrm{t}_{0}\right)$ lokalkompakt.

Bemerkung: Die hier betrachtete feinste Limitierung eines Vektorraumes tritt, wenn auch urbenannt, in Arbeiten von F.E.Browder et al. bei der Definition von endichstetigen Operatoren auf. Die endlichstetigen operatoren sind in reflexiven Banach-Räumen definierte operatoren, die stetic sind, wenn man den Definitionsbereich mit der von der feinsten Vektorraumimitierung induzierten Konvergenzstruktur und den Bilabereich mit der von der schwachen Topologie induzierten Topologie versieht. (5.6) Bemerkung: Man könnte nun vermuten, das die Iimjtierung to die einzige separierte vektorrauminitierung auf einem unendichdimensionalen vektorraum ist, mit der 
der. Vektorram lokalkompakt ist. Ein hier nur angedeutetes Beispiel zeigt, dak es noch weitere lokalkompakte Vektorraumlimitierungen auf. umendichoimensionalen vektorräumen gibt: Sej (E, $|\cdot|$ ) ein unendichdjmensionaler, reflexiver Banach-Raum. Mur jede naturliche zahl $n$ sej $S(0, n]$ die abgeschlossene Kugel um den Nulpunkt mit dem Radius n . $\sigma$ sei die schwache Topologie von $E$ bezüglich (E, $|\cdot|)$. Da $(E,|\cdot|)$ reflexiv ist, ist $\left(S(0, n], o_{S(0, n]}\right)$ ein kompakter, topologischer Raum. Es ist unmittelbar ersichtlich, dab zusammen mit den in natüricher weise gegebenen Einbettungsabbildungen die Familie $\left(\left(S(0, n], \dot{\sigma}_{S(0, n]}\right)_{n \in N}\right.$ ein induktives system kompakter, topolugischer Riame bildet. Mengentheoretisch ist der jnduktive Limes dieses systems der Vektorraum $E \cdot \operatorname{Sei}(E, \tau)=\operatorname{ind}_{N}(L)\left(S(0, n] ; \sigma_{S}(0, n]\right)$. Dann ist nach $(5.3)(E, \tau)$ ejn lokalkompakter Iimesraum. Man rechnet nun leicht die folgende äquivalente Definition der Limitierung $\tau$ nach:

Ein Filter $\theta$ auf $E$ konvergiert genau dann bezüglich $\tau$ gegen ein Element $x$ aus $E$, wenn er bezüglich $\sigma$ gegen $x$ konvergiert una zusätzlich eine beschränkte Menge enthält. Diese Definition wiederum ermöglicht es, ohne Schwierigkeiten zu verifizieren, daß $\tau$ eine Vektorramlimitierung ist. $(E, \tau)$ ist also ein lokalkompakter Iimesvektorraum. Da nach Konstruktion $\tau$ feiner als die separierte schwache Topologie $\sigma$ ist, folgt, daß $(E, \tau)$ separjert ist.Da ferner jeder bezügich der Norm konvergente Hilter auf $\mathrm{E}$ auch in der schwachen Topologie konvergiert und auBerdem stets beschränkte vengen enthäl, folgt, daß $\mathrm{t}$ grober als die Normtopologje sejn muß. Da E nach voraussetzung unendideh- 
dimensional ist, ist die Normtopologie stets echt grober a.ls die feinste vektorrauminitiemg von. $E$, da es bezüglich der Normtopologie unstetige lineare Funktionale gibt; bezüglich der feinsten Vektorraumimitierung muß abei nach deren unjersellex charakterisierung jedes lineare Funktional auf E stetig sein. Folglich ist die Normtopologie und somit auch $T$ echt gröber als die feinste Vektorraumimitierung.

\section{Literaturverueichris.}

[1] E. Binz:"Bemerkungen zu limitierten Funktionenalgebren." Math. Annalen 175,1968 , pp: $169-184$.

[2] W. Bucher:"Différentiabilité de la compositión et conplétitude de certains espaces fonctionnels." Comm. Math. HeIv. 43,1968 , pp. $256-288$.

[3] H.R. Fischer: "Limesräume." Math. Amalen 137, 1959, pp. $269-303$

[4] A. Fröljcher und W. Bucher:"Calculus in vector spaces without nom." Lecture Notes in Mathernatics 30 , BerinHeidelberg-New York, 1967 .

[5] H. Jarchow: Marinescu-Rëume" Comm. Math. Helv. 44, 1969, pp. $138-163$.

[6] H. Jarchow: "Dualität und Marinescu-Räume." Math. Annalen 182, pp. $134-144$.

[7] G. Köthe:" Topologische Iireare Räume I." Grundaehren der Mathematischen Wissenschaften in Einzeldarstellungen 107 " Berin-Hejdelberg-New York, 1966.

[8] K. Kutzler: "ijine Bernerkung uber endichaimensionale, sepa" 
rierte, limitiente Vektorräume." Arch. d. Math. 20, 1969. pp. $165-168$.

[9] F.T.M. Schroder: Dissertation, Queen's University, Kingston.

[10] H. Schubert: "Topologie". Teubner-Ver]ag, Stuttgart, 1264. 